\title{
Unconstrained Summoning for relativistic quantum information processing
}

\author{
Adrian Kent \\ Centre for Quantum Information and Foundations, DAMTP, Centre for Mathematical Sciences, \\ University of Cambridge, Wilberforce Road, Cambridge, CB3 OWA, U.K. and \\ Perimeter Institute for Theoretical Physics, 31 Caroline Street North, Waterloo, ON N2L 2Y5, Canada.
}

(Dated: December 18, 2018)

\begin{abstract}
We define a summoning task to require propagating an unknown quantum state to a point in space-time belonging to a set determined by classical inputs at points in space-time. We consider the classical analogue, in which a known classical state must be returned at precisely one allowed point. We show that, when the inputs are unconstrained, any summoning task that is possible in the classical case is also possible in the quantum case.
\end{abstract}

\section{INTRODUCTION}

In the not necessarily distant future, many or most significant economic decisions may depend on algorithms that process information arriving around the world as efficiently and quickly as possible, given light speed signalling constraints. Such algorithms will need to decide not only whether to make a trade, but also when and where. Quantum money enables efficient transactions, by allowing a delocalized quantum money token effectively to be "summoned" to a given space-time point as a result of information distributed through space-time. There are also alternative technological solutions [1] in the form of token schemes that do not require long term quantum state storage and in some embodiments are based entirely on classical communication.

This paper defines types of summoning task that naturally arise in relativistic economies, and shows that for an important and natural class of these tasks the two technologies have the same functionality. That is, if quantum money can be summoned to the correct space-time point in a given scenario, then so can classical tokens, and conversely. This has implications for the foundations of relativistic quantum theory, since summoning has proved a very fruitful way of understanding the properties of quantum information in space-time [2-5] and we also discuss these.

\section{SUMMONING}

Summoning [2, 3] is a task defined between two parties, Alice and Bob, who each have networks of collaborating agents. Each party trusts their own agents but not those of the other party. Summoning tasks discussed in the literature to date take the following form. In some fixed background space-time, Bob creates a quantum state (say, a qudit) in pre-agreed physical form, keeping its classical description private among his agents. One of his agents gives the state to one of Alice's at some point $P$. At one of a number of pre-agreed later points $\left\{c_{i}\right\}_{i \in I}$, Bob's local agent may ask for the state back from Alice's local agent. The pre-agreed set $I$ may be finite or infinite; the original discussion of the task [2] allowed $I$ to contain every point in the future of $P$ and also considered the possibility that $I$ contains just two space-like separated points.

Alice must then return the state at some point or within some region related to $c_{i}$ in a pre-agreed way. For example, in one version considered in Ref. [3], a request requires the state to be returned at the same point in space at a slightly later time, with respect to a given frame. In Ref. [4], this was generalized so that a request at $c_{i}$ requires the state to be returned at some pre-agreed point $r_{i}>c_{i}$. We say such a summoning task is possible in relativistic quantum theory if there is an algorithm that guarantees that Alice will comply with any allowed request (assuming she has ideal devices and instantaneous computing operations). Alice is allowed to return only a single state (here a qudit), so that compliance means guaranteeing that this is the state originally received.

Another interesting version of summoning allows any non-zero number of valid return points [5]. In this case we say the task is possible if there is an algorithm that guarantees that Alice will return the state to some valid return point. For example, one can consider a variant of Hayden-May's version of summoning in which requests may be made at any non-zero number of call points $\left\{c_{i}\right\}_{i \in I^{\prime}}$, where $I^{\prime} \subseteq I$, and the state must be returned at one of the corresponding return points, i.e. at some $r_{i}$ where $i \in I^{\prime}$. Interestingly, this is a strictly harder version of the task [5].

\section{EXTENDING THE DEFINITION OF SUMMONING}

For definiteness, we focus on the case of Minkowski space-time, which is a good local approximation in the region of the solar system. All the variants of summoning considered to date [2-5] are examples of a general class of quantum tasks in Minkowski 
space-time [3], in which Alice receives some number of classical and/or quantum inputs at a set of points in space-time and must produce classical and/or quantum outputs at another (possibly identical or overlapping) set of points, where the output points and information depend in some prescribed way on the input points and information. For the summoning tasks considered in Refs. [4, 5], the classical information consists of single bits ( 1 or 0 corresponding respectively to "call" or "no call") at a finite set of "call points" $\left\{c_{i}\right\}_{i=1}^{n}$, with a corresponding set of "return points" $\left\{r_{i}\right\}_{i=1}^{n}$. An input 1 at $c_{i}$ gives the instruction that returning the state at $r_{i}$ is required [4] or is one of the required set of possibilities [5]. The summoning tasks considered in Refs. [2, 3] also have single bit inputs. However, more general types of input and more general rules for defining allowed return points are also interesting. The key feature common to all summoning tasks considered to date is that an unknown quantum state is supplied and must be returned at some later point belonging to a set that is identified by information supplied during the task. It is natural to extend the term summoning to cover all such tasks.

We focus here on the case in which all the information supplied, other than the original unknown state, is classical, the sets of input points and possible return points are both finite and known in advance, and finite bounds on the information to be supplied at each input point are also known in advance. Our terminology here is to be understood modulo these restrictions, so that we speak here of "summoning" rather than "finite-input-point bounded-classical-input finite-output-point summoning". This is purely for brevity: more general types of summoning are also interesting. The bounds on classical inputs are imposed to simplify the notation; our results extend to general classical inputs, including unbounded integer or real number inputs.

We thus define a summoning task with one return point in a given space-time to be a task set by one party, Bob, for another party, Alice, who has an arbitrarily dense network of collaborating agents. Bob gives Alice (i.e. Bob's local agent gives Alice's local agent) an unknown quantum state at the start point $P$. Alice must produce the state at some return point $Q>P$. Here $Q \in\left\{Q_{1}, \ldots, Q_{N}\right\}$. Its identity depends, according to pre-agreed rules, on classical information supplied at some set of input points $\left\{P_{i}\right\}_{i=1}^{M}$. The point $P$ and the sets $\left\{P_{i}\right\}$ and $\left\{Q_{i}\right\}$ are known to both parties in advance of the task. The sets may overlap, and may include $P$. We take the sets of input and return points to be finite, although the infinite case is also interesting. The classical information Bob supplies at $P_{i}$ is an integer $m_{i}$ in the range $0 \leq m_{i} \leq\left(n_{i}-1\right)$. Alice must return the state to a point $Q\left(m_{1}, \ldots, m_{M}\right) \in\left\{Q_{1}, \ldots, Q_{N}\right\}$. Alice knows the functional dependence of $Q$ and the set $\left\{n_{i}\right\}_{i=1}^{M}$ in advance of the task; she learns the values of the $m_{i}$ only at the points $P_{i}$. To exclude trivial cases that complicate the notation, we assume that every return point may be designated by some set of inputs: i.e. for each $i$ there is at least one set of allowed inputs $m_{1}, \ldots, m_{M}$ such that $Q_{i}=Q\left(m_{1}, \ldots, m_{M}\right)$. We also assume that every input takes more than one possible value, i.e. that $n_{i} \geq 2$ for each $i$. We say the task is possible if, given unlimited predistributed classical and/or quantum resources, ideal devices and instantaneous classical and quantum computational power, there is an algorithm by which Alice can guarantee to return the state to $Q\left(m_{1}, \ldots, m_{M}\right)$ for any allowed set of inputs $\left\{m_{1}, \ldots, m_{M}\right\}$.

We define a summoning task with at most one return point similarly, allowing that $Q\left(m_{1}, \ldots, m_{M}\right)$ may be any point in the pre-agreed set $\left\{Q_{i}\right\}_{i=1}^{N}$ or may be the empty set $\varnothing$, in which case the state should not be returned at any point. Such tasks are possible only if there is an algorithm by which Alice can guarantee that the state is returned to a point $Q_{i}$ if and only if $Q_{i}=Q\left(m_{1}, \ldots, m_{M}\right)$, and is not returned anywhere if $Q\left(m_{1}, \ldots, m_{M}\right)=\varnothing$.

We may also define a summoning task with multiple return points, for which $Q\left(m_{1}, \ldots, m_{M}\right)$ may be any subset of $\left\{Q_{i}\right\}_{i=1}^{M}$, and $\left|Q\left(m_{1}, \ldots, m_{M}\right)\right|>1$ for at least one set of inputs.[12] Here we assume that for each $i$ there is at least one set of allowed inputs $m_{1}, \ldots, m_{M}$ such that $Q_{i} \in Q\left(m_{1}, \ldots, m_{M}\right)$. Such tasks are possible only if the algorithm guarantees that the state is returned to a point $Q_{i} \in Q\left(m_{1}, \ldots, m_{M}\right)$ if and only if $Q\left(m_{1}, \ldots, m_{M}\right)$ is non-empty, and is not returned anywhere if $Q\left(m_{1}, \ldots, m_{M}\right)=\varnothing$.

We distinguish between summoning tasks with constrained inputs and those with unconstrained inputs. In the former, Alice is guaranteed that some non-trivial constraint holds on the possible inputs. That is, there is at least one set of inputs $\left\{m_{1}, \ldots, m_{M}\right\}$, in the prescribed ranges $0 \leq m_{i} \leq\left(n_{i}-1\right)$, that is guaranteed never to arise. Hayden-May [4] define a summoning task with constrained inputs, since only one 1 input is allowed. The version of multi-call summoning [5] in which it is also allowed that no call may be made at any call point is a task with unconstrained inputs.

\section{CLASSICAL SUMMONING TASKS}

It is also interesting to consider classical versions of these summoning tasks. Here Alice is given a classical state at point $P$. We assume she can perfectly determine its classical description instantaneously, broadcast the description everywhere at light speed, and reconstruct a perfect copy instantaneously anywhere she receives the broadcast description. She thus may make arbitrarily many perfect copies of the state at $P$ or anywhere within its causal future. At each point $Q_{j}(j \in J)$ she must declare if she is returning the state; if she does, she must supply a copy of it. We assume she only has classical resources. We say the task is classically possible if there is an algorithm which guarantees that she will return a copy of the state at precisely one valid return point (if there are any) and at no other point. (So, if there are no valid return points, she does not return the state anywhere.) 


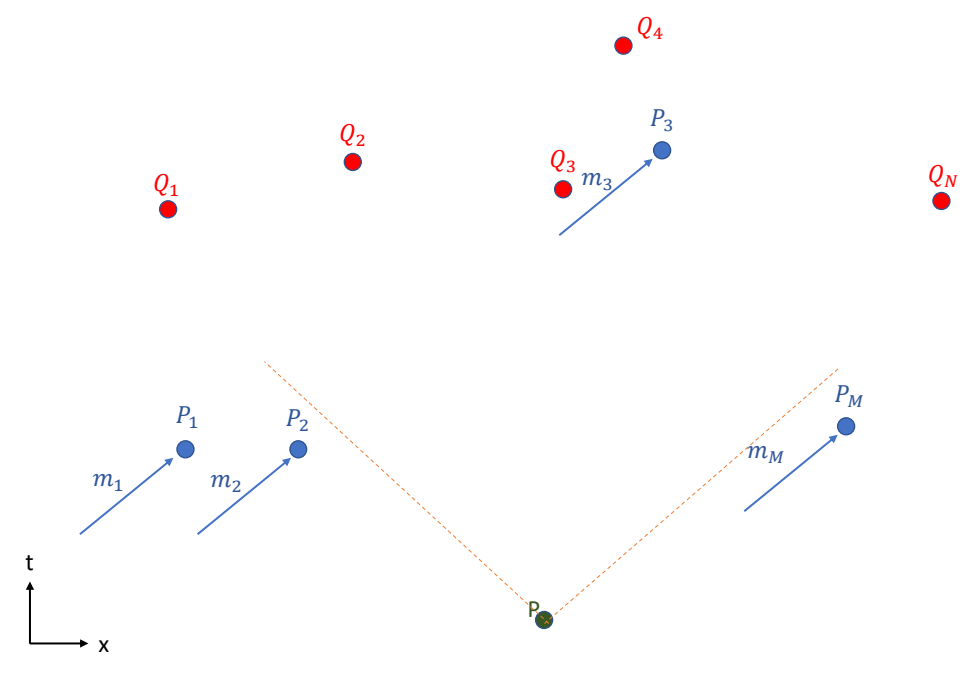

FIG. 1: A summoning task with at most one return point. Alice is given an unknown quantum state at the point $P$ and classical data in the form of integers $m_{i}$ at the points $P_{i}$. The dashed lines indicate the future light cone of $P$. She is required to send the quantum state to $Q\left(m_{1}, \ldots, m_{M}\right) \in\left\{Q_{1} \ldots Q_{M}\right\}$, or to retain it if $Q\left(m_{1}, \ldots, m_{M}\right)=\varnothing$.

Not all classically possible summoning tasks are also possible when a quantum state is being summoned. For example, in the original setting for the no-summoning theorem [2], Alice knows that precisely one call will be made, but does not know where. If she is required to return a classical state, she may simply broadcast its description everywhere, and return it in response to the call, wherever it is made. The same is true of Hayden-May summoning [4]. So long as all the return points are in the causal future of the start point, and the call points are in the causal past of each return point, Alice knows at each return point whether or not the state should be returned. Again, she may simply broadcast the description of the classical state to all the return points, and return it in response to the call, regardless of the relationship between the causal diamonds defined by the call and return points. In both cases, the task is thus classically possible, but quantum summoning is not generally possible.

These examples show that classical summoning may be possible while quantum summoning is impossible for a summoning task with constrained inputs. We now focus on summoning tasks with unconstrained inputs.

\section{SUMMONING TASKS WITH UNCONSTRAINED INPUTS AND AT MOST ONE RETURN POINT}

Consider now a classically possible summoning task with unconstrained inputs and at most one return point.

Lemma 1. Each return point $Q_{i}$ is in the causal future of the start point $P$, i.e. $Q_{i} \geq P$ for each $i$.

Proof. Each return point $Q_{i}$ may be designated by some set of allowed inputs. If $Q_{i}$ is so designated, Alice must propagate the qudit from $P$ to $Q_{i}$. Unless $Q_{i} \geq P$, this would require superluminal signalling.

Lemma 2. For every pair of return points $\left(Q_{i}, Q_{j}\right)$ the set of common past input points $S_{i j}=\left\{P_{k}: P_{k} \leq Q_{i} \& P_{k} \leq Q_{j}\right\}$ is non-empty.

Proof. Both $Q_{i}$ and $Q_{j}$ are designated return points for some (different) sets of inputs. Since the task is classically possible, the inputs at points in $S_{i}=\left\{P_{k}: P_{k} \leq Q_{i}\right\}$ must determine whether or not $Q_{i}$ is a (and hence the) valid return point. Since both $Q_{i}$ and $Q_{j}$ are valid return points for some sets of inputs, and the task is classically possible, $S_{i j}$ cannot be empty, otherwise some sets of inputs on $S_{i}$ and $S_{j}$ would be consistent with both $Q_{i}$ and $Q_{j}$ being valid return points.

Lemma 3. For every pair of return points $Q_{i}, Q_{j}$, any possible set of inputs at their common past input points $S_{i j}$ must logically exclude at least one of the pair as the designated return point.

Proof. If there is a set of inputs at points in $S_{i j}$ that is consistent with both $Q_{i}$ and $Q_{j}$ being a valid return point, these inputs must form part of a complete set of inputs that is consistent with both $Q_{i}$ and $Q_{j}$ being valid return points, since the inputs are 
unconstrained and it must be knowable at each $Q_{k}$ whether $Q_{k}$ is a valid return point. This contradicts the assumption of at most one valid return point.

The next result relies on the technique of distributed non-local computation [6], which relies on using pre-shared entanglement to implement a series of teleportation operations, with the classical teleportation data broadcast so that it is available to reconstruct the required quantum state at the appropriate point.

In the simplest example, Alice is given a quantum state $\psi$ at point $P$ and inputs $m_{1}, m_{2}$ at points $P_{1}, P_{2}$ respectively, and is required to return $\psi$ at point $Q\left(m_{1}, m_{2}\right) \in\left\{Q_{1}, Q_{2}\right\}$, where the function $Q\left(m_{1}, m_{2}\right)$ is known in advance. Suppose that $Q_{i} \geq P$ and $Q_{i} \geq P_{j}$ for $i=1,2$ and $j=1,2$. She may then carry out a teleportation operation on $\psi$ at $P$ using a predistributed entangled state shared between $P$ and (say) $P_{1}$, broadcasting the classical teleportation data. This produces a quantum state $\rho_{1}$ at $P_{1}$ from which $\psi$ may later be reconstructed. Now suppose that Alice has pre-shared $n_{1}$ labelled entangled states between $P_{1}$ and $P_{2}$. At $P_{1}$, she carries out a teleportation operation on $\rho_{1}$, using the entangled state with label $m_{1}$, and broadcasts the classical teleportation data and the value of $m_{1}$. At $P_{2}$, she sends the entangled state with label $m$ to the point $Q\left(m, m_{2}\right)$, and broadcasts the value of $m_{2}$. At $Q_{1}$ and $Q_{2}$, she receives both $m_{1}$ and $m_{2}$, and so knows which is the required return point. She also receives all the relevant teleportation data and the required quantum state at that return point, and so can reconstruct and return $\psi$ there.

By iterating this technique, we obtain the next lemma. This requires a large amount of pre-shared entanglement in examples with many call points; we are concerned here only with feasibility in principle, rather than resource optimization.

Lemma 4. If a state $\psi_{i j}$ is initially located at the start point $P$, it may be propagated in such a way that it arrives at $Q_{i}$ if the inputs on $S_{i j}$ preclude $Q_{j}$ (but not $Q_{i}$ ) as a valid return point and at $Q_{j}$ if the inputs on $S_{i j}$ preclude $Q_{i}$ (but not $Q_{j}$ ).

Proof. A non-local computation [6], taking into account the inputs at all points $P_{k} \in S_{i j}$, can be carried out by iterative teleportations from $P$ to one of these points and through a complete sequence of the remaining points, such that the teleportation output containing the quantum information linked with $\psi_{i j}$ is propagated appropriately to $Q_{i}$ or $Q_{j}$. We have that $P \leq Q_{i}$ and $P_{k} \leq Q_{i}$ for all $P_{k} \in S_{i j}$, and similarly for $Q_{j}$. The state may thus be reconstructed at the relevant return point from the classical teleportation data.

This algorithm could be extended to give rules as to where the state goes if both $Q_{i}$ and $Q_{j}$ are precluded; however this is irrelevant for our purposes.

The next lemma uses techniques of and results about quantum secret sharing, developed in Refs. [7, 8]. A quantum secret sharing scheme for quantum states in a given Hilbert space $H$ is defined by a quantum operation $A: H \rightarrow H_{1} \otimes \ldots \otimes H_{n}$ from $H$ to a tensor product of component Hilbert spaces $H_{i}$, together with quantum operations $A_{S}: \otimes_{i \in S} H_{i} \rightarrow H$ for some subsets $S \subseteq\{1, \ldots, n\}$, such that $A_{S} \cdot A=I$. The access structure of the secret sharing scheme is the list $L$ of subsets $S$ for which such an operation $A_{S}$ is defined. Effectively, a quantum secret sharing scheme allows an unknown quantum state $\psi$ to be shared among $n$ parties in such a way that any subset $S$ of them belonging to the access structure may reconstruct $\psi$. Quantum secret sharing schemes can be constructed with any access structure that is monotonic (so that if $S_{1} \in L$ and $S_{1} \subseteq S_{2}$ then $S_{2} \in L$ ) and does not violate the no-cloning theorem (so that if $S_{1} \cap S_{2}=\varnothing$ and $S_{1} \in L$ then $S_{2} \notin L$ ). Here again we are presently interested only in whether a scheme exists in principle; we do not consider resource optimization.

Lemma 5. There is a quantum secret sharing scheme for a general state $\psi$, with components labelled by $\left\{\psi_{i j}\right\}_{1 \leq i<j \leq N}$, with the property that $\psi$ may be reconstructed from any subset of the form $\left\{\psi_{i j}\right\}_{1 \leq j \leq N ; j \neq i}$, where we set $\psi_{j i}=\psi_{i j}$ if $j>i$.

Proof. No pair of these subsets are disjoint, and so they satisfy the conditions to generate an access structure for a quantum secret sharing scheme [7, 8].

Theorem 1. A classically possible summoning task with unconstrained inputs and at most one return point is also a possible quantum summoning task.

Proof. This follows by construction from the preceding lemmas.

\section{SUMMONING TASKS WITH UNCONSTRAINED INPUTS AND MULTIPLE RETURN POINTS}

Consider now a classically possible summoning task with unconstrained inputs and multiple return points. Since the task is classically possible, there must be some classical algorithm that allows Alice to decide, based on the inputs, to exclude returning at one of any pair $Q_{i}$ and $Q_{j}$ of return points when both are valid. Without loss of generality, we may assume this algorithm is deterministic, since Alice has only classical resources, and any classical randomness in a probabilistic algorithm may be precomputed and predistributed. The algorithm must be consistent: i.e. it must identify a valid return point when there is one. The determination of whether $Q_{i}$ or $Q_{j}$ is excluded can only depend on the inputs at points in $S_{i j}$, by causality. 
We can thus incorporate the algorithm within a refined definition of the task, producing a summoning task with unconstrained inputs and at most one return point. We may delete any return points that are never used by the algorithm. This defines a possible quantum summoning task, by the previous discussion. Hence we have:

Theorem 2. A classically possible summoning task with unconstrained inputs and multiple return points is also a possible quantum summoning task.

\section{CLASSICAL AND QUANTUM POSSIBILITY}

We have shown that classically possible summoning tasks with unconstrained inputs are also possible quantum summoning tasks, whether they have at most one return point or multiple return points.

Conversely, consider a quantum summoning task that can be solved with a deterministic quantum algorithm, by which we mean an algorithm that always returns the state to the same return point $Q\left(m_{1}, \ldots, m_{M}\right)$ for a given set of inputs $m_{1}, \ldots, m_{M}$. By including appropriate ancillae, we can describe any such quantum algorithm as a deterministic sequence of unitary operations, in which unitaries $U_{Q}$ act on the collective state at point $Q$ and propagate outputs along secure channels to further points $R_{i}>Q$. All the operations that might be performed in this algorithm can be described classically: "apply unitary $U_{Q}$ at $Q$ ", "prepare state $\phi$ at $Q^{\prime \prime}$, and so on, where each operation depends in a prescribed way on inputs received at the relevant point or in its causal past. If the algorithm is deterministic in the sense above, then the success of a summoning task that is supposed to propagate a state from the start point $P$ to a valid return point $Q_{j}$ is determined by, and deducible from, the subset of these operations applied in the past light cone of $Q_{j}$.

Hence any deterministic quantum algorithm that guarantees success may be simulated by classical communications, describing the relevant operations and state preparations, broadcast from the corresponding space-time points. This broadcast simulation allows Alice's agent at the valid return point $Q_{j}$ (if there is a valid return point), to deduce that the quantum algorithm would have returned the quantum state there, and thus that she should return a copy of the classical state there. It also allows Alice's agents at all other return points $Q_{k} \neq Q_{j}$ to deduce that the quantum algorithm would not have returned the quantum state at their locations, and hence that they should not return copies of the classical states. This gives us:

Theorem 3. A quantum summoning task with unconstrained inputs and multiple return points that can be solved with a deterministic quantum algorithm is also classically possible.

\section{DISCUSSION}

Summoning can be thought of as a type of distributed quantum computation, or more generally a sub-routine within such a computation, in which quantum states need to be propagated in response to incoming classical information. This information could come from nature (for example detected photon fluxes near given spacetime points), from human activity (for example local market prices at given points in time on a distributed financial network), or as outputs from other computations or other parts of the same computation (which may themselves use natural and/or human-made inputs).

Our results further illustrate (cf. [3, 6, 9-11]) the power of quantum information in a relativistic context. Roughly speaking, they show that if a classical observer at a given spacetime point can know, from available classical data that has no known constraints, that a given unknown quantum state should have been propagated to them, then there was an infallible algorithm that could have done so, and vice versa. We take this as further support for viewing summoning as a key primitive of relativistic quantum information theory.

The algorithm defined is almost certainly far from optimal for most interesting unconstrained summoning tasks. It would be very interesting to understand better how to optimize the use of entanglement and other resources for these tasks. One strong motivation for doing so comes from financial and other applications of distributed algorithms on networks where relativistic signalling constraints are significant. As noted earlier, token or money schemes that prevent illegitimate duplication can in principle be based on quantum money, but can also be implemented by alternative techniques that require no long term quantum state storage, and in some cases no quantum information processing at all [1]. Unconstrained summoning tasks are natural problems in this context: a market agent wants to be able to respond as flexibly and fast as possible to incoming market data across the network, and to be able to present their money token at the optimal point in space-time, according to some appropriate financial metric. An unconstrained quantum summoning scheme in which quantum money tokens are summoned and presented is an elegant theoretical solution. However if, as seems very plausible, such schemes generally require unfeasible amounts of entanglement to solve realistic problems, then rival technologies [1] are likely to prove advantageous. 
Acknowledgments This work was partially supported by UK Quantum Communications Hub grant no. EP/M013472/1 and by Perimeter Institute for Theoretical Physics. Research at Perimeter Institute is supported by the Government of Canada through Industry Canada and by the Province of Ontario through the Ministry of Research and Innovation.

[1] Adrian Kent. Summonable supermoney: virtual tokens for a relativistic economy. arXiv preprint arXiv:1806.05884, 2018.

[2] Adrian Kent. A no-summoning theorem in relativistic quantum theory. Quantum Information Processing, 12(2):1023-1032, 2013.

[3] Adrian Kent. Quantum tasks in Minkowski space. Classical and Quantum Gravity, 29(22):224013, 2012.

[4] Patrick Hayden and Alex May. Summoning information in spacetime, or where and when can a qubit be? Journal of Physics A: Mathematical and Theoretical, 49(17):175304, 2016.

[5] Emily Adlam and Adrian Kent. Quantum paradox of choice: More freedom makes summoning a quantum state harder. Physical Review A, 93(6):062327, 2016.

[6] Lev Vaidman. Instantaneous measurement of nonlocal variables. Physical Review Letters, 90(1):010402, 2003.

[7] Daniel Gottesman. Theory of quantum secret sharing. Physical Review A, 61(4):042311, 2000.

[8] Adam D Smith. Quantum secret sharing for general access structures. arXiv preprint quant-ph/0001087, 2000.

[9] Adrian Kent. Unconditionally secure bit commitment with flying qudits. New Journal of Physics, 13(11):113015, 2011.

[10] Adrian Kent, William J Munro, and Timothy P Spiller. Quantum tagging: Authenticating location via quantum information and relativistic signaling constraints. Physical Review A, 84(1):012326, 2011.

[11] Harry Buhrman, Nishanth Chandran, Serge Fehr, Ran Gelles, Vipul Goyal, Rafail Ostrovsky, and Christian Schaffner. Position-based quantum cryptography: Impossibility and constructions. SIAM Journal on Computing, 43(1):150-178, 2014.

[12] A fully exhaustive terminology would again distinguish between the cases where $Q\left(m_{1}, \ldots, m_{M}\right)$ is empty for at least one set of inputs and where it is never empty. We will not need to discuss these separately here. 